\title{
Noise-induced bursting in stochastic models of single cells and electrically coupled ensembles
}

\section{Georgi Medvedev}

Address: Department of Mathematics, Drexel University, Philadelphia, PA 19104

Email: Georgi Medvedev - medvedev@drexel.edu

from Seventeenth Annual Computational Neuroscience Meeting: CNS*2008

Portland, OR, USA. 19-24 July 2008

Published: II July 2008

BMC Neuroscience 2008, 9(SuppI I):O5 doi:I0.1 I86/I47I-2202-9-SI-O5

This abstract is available from: http://www.biomedcentral.com/I47I-2202/9/SI/O5

(c) 2008 Medvedev; licensee BioMed Central Ltd.

Bursting is a common form of electrical activity in individual neurons and neuronal networks characterized by alternating phases of spiking and quiescence. Many physiological and cognitive functions of the nervous system rely on the rhythms generated by bursting oscillations. In particular, bursting plays a prominent role in central pattern generators controlling various types of rhythmic behaviors such as respiration, heartbeat, and locomotion to name a few. In many cases, bursting results from the slow modulation by ionic currents with slow gating or slowly changing calcium concentration. Therefore, slow-fast systems of differential equations provide a natural framework for studying dynamical mechanisms of bursting [1]. The slow-fast analysis of bursting reveals the relation between the structure of the differential equation model (more precisely, the bifurcation structure of the fast subsystem) and the qualitative features of the bursting patterns that it produces. This relation can be used to explain dynamical mechanisms underlying bursting in variety of deterministic conductance-based models $[1,2]$.

However, many neural systems are subject to noise. There are multiple sources of noise such as temperature fluctuations, stochastic character of synaptic transmission and stochastic channel fluctuations, which can affect the firing patterns of neurons. Therefore, it is important to identify and characterize effects of noise on the patterns of neural activity. In systems with multiple stable states, such as conductance-based models of bursting neurons, noise may induce transitions between different attractors in the system dynamics, thus creating qualitatively new dynamical regimes, which are not present in the deterministic system. In this work, we systematically study this situation for a class of square-wave bursting models of excitable cell membranes [3]. This class includes many conductancebased models of neural and endocrine cells such as neurons in the Pre-Botzinger Complex, pancreatic beta-cell, and certain pyramidal neurons. Furthermore, we extend our analysis to networks of electrically coupled bursting cells and demonstrate that gap junction coupling can produce unexpected effects on the system dynamics in the presence of noise. In summary, the present study contributes to elucidating the role of noise in shaping patterns of electrical activity in neural cells and electrically coupled networks.

\section{Acknowledgements}

This work was partially supported by NSF grant IOB 0417624 .

\section{References}

I. Rinzel J: A formal classification of bursting mechanisms in excitable systems. In Proceedings of the International Congress of Mathematicians Edited by: Gleason AM. AMS; 1987:135-169.

2. Izhikevich EM: Dynamical Systems in Neuroscience: The Geometry of Excitability and Bursting. The MIT Press, Boston, MA; 2007.

3. Hitczenko P, Medvedev GS: Bursting oscillations induced by small noise. [http://arxiv.org/abs/07/2.4074]. arXiv:07/2.4074 